\title{
Farmacovigilância: um passo em direção ao uso racional de plantas medicinais e fitoterápicos
}

\author{
Evelin E. Balbino, ${ }^{*}, 1$ Murilo F. Dias ${ }^{2}$ \\ ${ }^{1}$ Agência Nacional de Vigilância Sanitária, Gerência Geral de Medicamentos, SIA, Trecho 5, Área Especial 57, \\ 71205-050 Brasilia, DF, Brasil, \\ ${ }^{2}$ Agência Nacional de Vigilância Sanitária, Gerência de Farmacovigilância, SIA, Trecho 5, Área Especial 57, \\ 71205-050 Brasília, DF, Brasil.
}

\begin{abstract}
RESUMO: Neste artigo são apresentados os resultados da avaliação das notificações de eventos adversos a plantas medicinais e seus derivados (fitoterápicos), efetuadas voluntariamente ao Sistema Nacional de Farmacovigilância, coordenado pela Agência Nacional de Vigilância Sanitária, no período de janeiro de 1999 a março de 2009. A farmacovigilância envolve a detecção de reações adversas que ocorrem durante ou após o uso de um medicamento, a interação medicamentosa, o desvio de qualidade, o uso abusivo e a inefetividade. Como as pesquisas realizadas para a avaliação do uso eficaz e seguro de fitoterápicos são incipientes, as notificações de eventos auxiliam na geração de novas informações, promovendo seu uso racional.
\end{abstract}

Unitermos: Fitoterápicos, plantas medicinais, farmacovigilância, evento adverso, Anvisa.

\begin{abstract}
Pharmacovigilance: a step towards the rational use of herbs and herbal medicines". This article presents the evaluation of the notifications of adverse events related to medicinal plants and their derivatives (herbal medicines). Such evaluations were voluntarily run by the Brazilian Pharmacovigilance System, coordinated by the Brazilian Health Surveillance Agency (Anvisa), from January 1999 to March 2009. Pharmacovigilance involves the detection of adverse reactions that occur during or after the use of drugs, drug interactions, problems of quality, improper use and inefficacy. There is scarce scientific confirmation about efficacy and safety of herbal medicines, so the notification of adverse events provides of information, and promotes rational use.
\end{abstract}

Keywords: Herbs, herbal medicines, pharmacovigilance, adverse events, Anvisa.

\section{INTRODUÇ̃̃O}

O uso de plantas medicinais é amplamente difundido e encontra-se em expansão pelo mundo (WHO, 2004). No Brasil, recentemente, foi publicada a Política Nacional de Práticas Integrativas e Complementares no Sistema Único de Saúde (SUS), que visa ampliar as opções terapêuticas oferecidas aos usuários do SUS, com garantia de acesso a plantas medicinais, fitoterápicos e outros serviços relacionados, com segurança, eficácia e qualidade (Ministério da Saúde, 2006a).

A toxicidade de medicamentos preparados com plantas pode parecer trivial, quando comparada com os tratamentos convencionais, entretanto é um problema sério de saúde pública. Plantas medicinais podem desencadear reações adversas pelos seus próprios constituintes, devido a interações com outros medicamentos ou alimentos, ou ainda relacionados a características do paciente (idade, sexo, condições fisiológicas, características genéticas, entre outros). Erros de diagnóstico, identificação incorreta de espécies de plantas e uso diferente da forma tradicional podem ser perigosos, levando a superdose, inefetividade terapêutica e reações adversas (WHO, 2002). Além disso, o uso desses produtos pode comprometer a eficácia de tratamentos convencionais, por reduzir ou potencializar seu efeito (Capasso et al., 2000).

As pesquisas realizadas para avaliação do uso seguro de plantas medicinais e fitoterápicos no Brasil são incipientes, assim como o controle da comercialização pelos órgãos oficiais em feiras livres, mercados públicos ou lojas de produtos naturais (Veiga Junior et al., 2005). Muitas vezes ocorrem adulterações propositais e não declaradas com substâncias farmacêuticas potentes como corticóides, antidepressivos e anorexígenos. Efeitos adversos também podem advir da contaminação por agrotóxicos, metais pesados e microrganismos (WHO, 2004). Para registrar essas ocorrências, inclusive aquelas reações muito raras, mas severas, um sistema de coleta de 
dados e a organização, avaliação e posterior divulgação das informações coletadas é de extrema importância (Capasso et al., 2000).

Há uma dificuldade de identificar eventos adversos a plantas medicinais, tanto pelo usuário como por profissionais de saúde, porque não se faz uma correlação direta de seu uso ao sintoma desenvolvido. Sabe-se que na Inglaterra e nos Estados Unidos sub-notificações são significantes nos esquemas de notificação voluntária a medicamentos preparados com plantas, porque os usuários não procuram orientação médica para o seu uso e não relatam seus efeitos adversos (Bames, 2003; Cupp, 1999). No Brasil, a dispensação de plantas medicinais é privativa das farmácias e ervanarias, observados o acondicionamento adequado e a classificação botânica (Congresso Nacional, 1973). Além disso, plantas medicinais e seus derivados podem ser obtidos na forma de produtos manipulados, industrializados cadastrados como alimentos e cosméticos ou registrados como medicamentos fitoterápicos e medicamentos dinamizados. Atualmente, a Resolução RDC n ${ }^{0} 48$, de 16 de março de 2004, da Agência Nacional de Vigilância Sanitária (Anvisa), normatiza o registro de medicamentos fitoterápicos (Anvisa, 2004a) enquanto que a Resolução RDC $\mathrm{n}^{\circ} 4$, de 10 de fevereiro de 2009, dispõe sobre as normas de farmacovigilância (Anvisa, 2009a).

A Organização Mundial da Saúde (OMS) define o conceito de farmacovigilância como sendo a ciência relativa à detecção, avaliação, compreensão e prevenção dos efeitos adversos ou quaisquer problemas relacionados a medicamentos (WHO, 2002). A farmacovigilância visa detectar precocemente eventos adversos conhecidos ou não, monitorando também possíveis aumentos na incidência dos mesmos. O sistema deve ter a capacidade de avaliar os benefícios e riscos do produto, para assegurar que esse mantenha a qualidade, segurança e eficácia compatíveis com seu uso racional (Anvisa, 2009c). Desde 2001, o Brasil é membro do programa da OMS, coordenado pelo Centro Colaborador do Uppsala Monitoring Centre, para gestão da base de dados internacional das notificações de eventos adversos recebidos dos centros nacionais participantes (Mendes et al., 2008).

Até março de 2008, as notificações de eventos adversos que chegavam à Gerência de Farmacovigilância da Anvisa pelo formulário de notificação disponível no seu sítio eletrônico, eram avaliadas e armazenadas, de forma manual, no banco de dados denominado Bdfarm. Relatos de eventos adversos que chegavam à gerência por outros meios eram cadastrados no banco de dados Sisfarmaco. A partir desta data, com o intuito de receber informações qualificadas diretamente na forma de banco de dados, foi criado o Sistema Nacional de Notificações para a Vigilância Sanitária (Notivisa). Esse banco de dados recebe notificações de eventos adversos de profissionais de saúde ou de usuários cadastrados, por meio de formulários de notificação (Anvisa, 2009c). Os usuários podem também comunicar eventos adversos ao profissional de saúde ou para a Vigilância Sanitária Local, que deve, por sua vez, repassar essa informação à Anvisa.

O objetivo deste trabalho foi traçar um perfil das notificações de eventos adversos encaminhadas para a Anvisa sobre plantas medicinais e fitoterápicos. A divulgação dos dados obtidos visa sensibilizar os profissionais de saúde e usuários quanto ao risco sanitário associado ao uso indiscriminado desses produtos, além de promover a atualização das bulas de medicamentos fitoterápicos, permitindo uma melhor análise riscobenefício.

\section{MATERIAL E MÉTODOS}

A presente análise foi realizada por meio de avaliação retrospectiva nos bancos de dados da Gerência de Farmacovigilância da Anvisa: Bdfarm, Sisfarmaco e Notivisa. Foram selecionadas as notificações de eventos adversos que citavam plantas medicinais e fitoterápicos no período de janeiro de 1999 a março de 2009. Como critério de exclusão, foi adotado que medicamentos em fase de estudos clínicos ou compostos por substâncias isoladas, mesmo sendo provenientes de extratos vegetais, não seriam considerados parte do estudo. Em seguida, os eventos adversos selecionados foram comparados com dados publicados em livros recomendados na Lista de referências bibliográficas para avaliação de segurança e eficácia de fitoterápicos (Anvisa, 2004b) e outras publicações técnico-científicas. Nessa etapa, apenas foram incluídas notificações que continham, no mínimo, as informações sobre a nomenclatura botânica ou marca comercial do fitoterápicos suspeito e descrição do evento adverso. de quadro.

Os resultados obtidos foram descritos na forma

\section{RESULTADOS E DISCUSSÃO}

Os bancos de dados de Farmacovigilância da Anvisa possuem mais de vinte mil notificações de eventos adversos a medicamentos, acumulados no período proposto para o estudo. Aplicando-se o critério de inclusão, 71 notificações de eventos adversos tiveram plantas medicinais ou fitoterápicos como principal suspeito e seis como suspeito secundário, totalizando 77 notificações incluídas no estudo. Algumas notificações relatam mais de uma reação adversa, obtendo-se um total de 165 eventos adversos a fitoterápicos.

Os dados analisados permitem observar que a média das idades entre os pacientes constantes nas notificações avaliadas foi de 40,4 anos (DP 19,7). Houve variação entre 0 - 84 anos. O sexo prevalente foi o feminino com 47 notificações $(64,4 \%)$. Em duas notificações o sexo 
foi ignorado $(2,7 \%)$. Esta desproporção entre homens e mulheres já é conhecida na farmacovigilância, sendo que as mulheres são duas vezes mais propensas a reações adversas (Wiffen et al., 2002).

Os eventos adversos foram notificados por profissionais de saúde (51\%), por usuários $(32 \%)$ e em $17 \%$ das notificações não foi informada a origem. Dentre os profissionais de saúde, 58,8 \% das notificações são provenientes de farmacêuticos, $33,3 \%$ de médicos e $7,8 \%$ de enfermeiros. O grande número de notificações por parte dos usuários evidencia que a contribuição de consumidores ao sistema de farmacovigilância a plantas medicinais não pode ser menosprezada.

Voluntários foram responsáveis por $62 \%$ das notificações a eventos adversos, e hospitais sentinela por $17 \%$. A indústria, que repassa informações recebidas pelo Serviço de Atendimento ao Consumidor, contribuiu com $11 \%$. Outros 4\% foram transferidos à Anvisa a partir dos centros de vigilância sanitária dos Estados e Municípios.

Constatou-se que um terço das notificações de eventos adversos refere-se a plantas medicinais ou seus derivados sem registro na Anvisa, evidenciando a existência de uma ampla rede de distribuição de difícil controle, como feiras livres, mercados municipais, internet e correio, entre outros.

Todas as notificações recebidas pela Anvisa são avaliadas quanto a gravidade e a causalidade. Dentre as notificações selecionadas, trinta eventos adversos foram considerados graves $(18,3 \%)$, segundo a classificação CIOMS V (CIOMS, 2001), dentre os quais três levaram a óbito. Uma por aplasia medular (Garcinia cambogia) e outras duas por hepatite fulminante (Piper methysticum). A avaliação da causalidade para $G$. cambogia foi considerada possível. Nos casos dos óbitos relativos ao P. methysticum descartou-se a possibilidade de hepatite viral e alcoólica.

Houve uma notificação de uso abusivo, após o tratamento prolongado com um medicamento fitoterápico com alto teor de etanol. Outra notificação relatou "queimação na pele" imediatamente após o uso tópico e "dor no fígado" após uma semana de administração oral de um produto sem registro, com alto teor de etanol. Por ser um ótimo solvente, o etanol é muito usado para obtenção de derivados de plantas medicinais e para manter os ingredientes ativos dissolvidos em meio líquido. Esses produtos não devem ser usados por período prolongado e deve-se ter cautela ao usá-los em crianças, gestantes, lactantes, pacientes com distúrbios hepáticos, entre outros. A bula de medicamentos fitoterápicos e dinamizados deve incluir uma frase de advertência, com informação sobre o teor de álcool do medicamento (Anvisa, 2009b).

Os resultados demonstram a necessidade de fortalecimento do sistema de farmacovigilância de plantas medicinais e fitoterápicos no intuito de promover o seu uso racional. Após a publicação de dois Alertas Federais de Farmacovigilância pela Anvisa, em 2001 e 2002 (Anvisa, 2002a), para advertir contra as graves reações adversas associadas ao uso de extratos de P. methysticum, e a inclusão da advertência à possível hepatotoxicidade nas bulas, não houve mais notificações de eventos adversos a este medicamento à Anvisa, o que pode significar que os alertas tenham influenciado no perfil do uso do medicamento, aumentando sua segurança.

O Notivisa, banco de dados atualmente utilizado, foi elaborado para o recebimento de notificações por profissionais de saúde cadastrados, em formulários padronizados, com o objetivo de receber informações que caracterizem tecnicamente o evento adverso. Quando a notificação é feita diretamente pelo usuário, é necessário o cadastro e preenchimento de um formulário extenso, relatos provenientes por outros meios não são mais cadastrados no banco de dados. Desde a criação do Notivisa, não foram detectadas notificações de evento adverso a plantas medicinais e fitoterápicos, podendo demonstrar que o mesmo dificultou o acesso ao sistema de farmacovigilância de usuários desses produtos. Essa ferramenta de farmacovigilância não é ideal para plantas medicinais e seus derivados, que comumente são a primeira opção de tratamento, geralmente feito sem acompanhamento de profissional de saúde.

Os profissionais de saúde precisam ser treinados para questionar os pacientes sobre o uso de plantas medicinais e fitoterápicos e devem ser incentivados a notificar essas reações ao Sistema Nacional de Farmacovigilância. Os usuários devem buscar recomendações de uso com profissionais da saúde e procurar atendimento diante de qualquer suspeita de reação adversa. Este propósito poderá ser alcançado com a sensibilização dos profissionais de saúde, campanhas educativas, agilidade e constância na transferência das notificações existentes nos centros de vigilância dos Estados e Municípios à Anvisa, maior integração das áreas da Anvisa que regulamentam medicamentos (registro, inspeção e farmacovigilância), disponibilização dessas informações no sítio eletrônico da Anvisa e, quando necessário, inclusão de novos eventos adversos nas bulas dos medicamentos, publicação de alertas e inspeções em empresas fabricantes. A participação efetiva desses diversos atores, de forma harmônica, é fundamental para fortalecer o Sistema Nacional de Vigilância Sanitáris, com ênfase ao monitoramento de plantas medicinais e fitoterápicos comercializadas no país, visando o seu uso racional e norteando as ações regulatórias no mercado farmacêutico brasileiro, baseado no acúmulo de informações. 
Quadro 1. Relação entre as informações sobre eventos adversos a plantas medicinais no banco de dados da anvisa e dados publicados sobre estas plantas.

\begin{tabular}{|c|c|c|c|c|}
\hline Nome & Indicações & Notificações da farmacovigilância & $\begin{array}{l}\text { Relatos de eventos adversos na } \\
\text { literatura }\end{array}$ & Referência \\
\hline $\begin{array}{l}\text { Aesculus } \\
\text { hippocastanum }\end{array}$ & $\begin{array}{l}\text { Tratamento de varizes, } \\
\text { edemas dos membros } \\
\text { inferiores e hemorróidas. }\end{array}$ & $\begin{array}{l}\text { Uma notificação de inefetividade. } \\
\text { Uma notificação de eritema e } \\
\text { sudorese, outra de depressão e } \\
\text { angústia respiratória e uma terceira } \\
\text { de vômito, tontura, mal estar geral } \\
\text { e sudorese diminuída, em relação } \\
\text { a uma associação de rutina, A. } \\
\text { hippocastanum e miroton (extrato seco } \\
\text { de Adonis vernalis, Nerium oleander, } \\
\text { Scila maritima e Convallaria majalis). }\end{array}$ & $\begin{array}{l}\text { Relatos de pruridos, náusea, } \\
\text { cefaléia e tontura. } \\
\text { Há relatos de síndrome de } \\
\text { pseudolupus, com artralgias, } \\
\text { febre recorrente, mialgias, } \\
\text { manchas na pele e alterações } \\
\text { cardiorespiratórias para } \\
\text { um produto denominado } \\
\text { Venocuran (fenopirazona com } A \text {. } \\
\text { hippocastanum e miroton). }\end{array}$ & Anvisa, 2004b. \\
\hline
\end{tabular}

Allium cepa Antimicrobiano tópico e antitrombótico.

Ananas $\quad$ Expectorante.
comosus

Atropa

belladonna

Bauhinia
forficata

Borago

officinalis

Calendula officinalis

é fonte de ácido gamalinolênico. e antiinflamatório.
Uso sistêmico: ação anti-colinérgica parassimpaticolítica, espasmolítica e efeito dromotrópico e cronotrópico positivo.

Uso tópico: contra-irritante para alívio de dor.
Duas notificações de alargamento e hipertrofia da queimadura após o uso tópico de uma associação de A. cepa com alantoína e heparina sódica, indicada como cicatrizante. Uma notificação de irritação de pele e coceira intensa em relação ao mesmo produto.

Notificação de um caso de choque anafilático em paciente de 4 anos que tomou diclofenaco e um xarope de abacaxi. Em relação ao diclofenaco há várias notificações de reações alérgicas.

Duas notificações de hepatite, após uso oral de uma associação de acriflavina, metenamina, metiltionínio e $A$. belladonna, um anti-séptico das vias urinárias. Cada $15 \mathrm{mg}$ de extrato de $A$. belladonna, contém 0,045 mg de alcalóides hiosciamina-atropina. Uma notificação de taquicardia, midríase, mal estar geral e agitação após uso oral, por uma paciente que, concomitantemente à associação anterior, usou outro anticolinérgico, butilbrometo de escopolamina. Uma notificação de erupção cutânea e prurido, também em relação à associação anterior.

Duas notificações de efeitos anticolinérgicos sistêmicos, como midríase, taquicardia, hipertensão, vertigem, boca seca, falta de controle membros inferiores, em relação a uma associação de Hamamelis virginiana, Davilla rugosa, A. belladonna, mentol e lidocaína, para uso tópico em varizes e hemorróidas. Cada grama da pomada contém $40 \mathrm{mg}$ de extrato mole de $A$. belladonna com $0,5 \mathrm{mg}$ de alcaloides.

Quatro notificações de reações adversas graves, como problemas hepáticos, inclusive cirrose, e dor renal, envolvendo um medicamento comercializado sem registro.

Uma notificação de diarréia, eructação e inchaço abdominal.

Uso tópico como cicatrizante
Contato repetido com a droga vegetal raramente pode ocasionar reações alérgicas, como eczema das mãos.

Relatos de náuseas, vômitos, diarréias, reações alérgicas e menorragias. Hipersensibilidade cruzada a papaína e pólen de grama.

Relatos de rubor, boca seca, midríase, hipertermia devido a redução da sudorese, taquicardia, dificuldade de micção e obstipação. A janela terapêutica é estreita e a sobredosagem leva a nervosismo, alucinação, delírio e coma.

$\mathrm{O}$ antibacteriano metenamina pode causar distúrbios gastrintestinais e é contra-indicado em pacientes com insuficiência hepática por liberar amônia no trato gastrintestinal.

Emplastros e linimentos de beladona têm sido usados como contra-irritantes para alívio de dor, porém há pouca evidência de seu efeito benéfico e efeitos colaterais têm ocorrido. A atropina, um alcalóide do grupo hiosciaminaatropina, é rapidamente absorvida pela mucosa e em parte pela pele intacta, induzindo efeitos sistêmicos.

Não há relatos de reações adversas na bibliografia consultada.

Anvisa, 2004b; Brinker, 2009.

Não há relatos de reações adversas. Anvisa, 2004b; O óleo da semente não contém alcalóides pirrolizidínicos.

Raramente ocorre dermatite de contato após o uso da tintura. Pode ocorrer sensibilização após contato freqüente com a droga vegetal.
Anvisa, 2004b;

Brinker, 2009.

Anvisa, 2004b;

Martindale, 1996.
Brinker, 2009.

Anvisa, 2004b.
Uma notificação de enxantema maculopapular após o uso tópico de uma associação de óleo de calêndula, óleo de amêndoa doce e óleo de macadâmia. 


\begin{tabular}{ll}
\hline Camellia & $\begin{array}{l}\text { Tratamento da fragilidade } \\
\text { capilar e alterações da } \\
\text { sinensis }\end{array}$ \\
& permeabilidade vascular.
\end{tabular}

Colocasia antiquorum

Convolvulus scammonia

Cynara scolymus

Ephedra sínica

Fucus vesiculosus

Garcinia cambogia

Laxante. Indicação alemã registrada.

Uso oral: Colagogo e colerético.

Coadjuvante no tratamento da obesidade relacionada à deficiência de iodo e hipotireoidismo.

Auxilia na regulação do apetite.
Não há dados na literatura técnico-científica consultada.
Quatro notificações de reações adversas como hipertensão e arritmia cardíaca, cefaléia, tremor, euforia e insônia, após o consumo de uma associação de C. sinensis, Malva silvestre, cardo e casca de limão, comercializada como suplemento alimentar. Há uma notificação de suspeita de hepatite tóxica, em relação a este mesmo produto. Para um produto, cujo registro foi cancelado, composto por uma associação de $C$. sinensis e Ortosiphon stamineus (chá-de-java), um hospital sentinela encaminhou uma notificação de hepatite medicamentosa.

Vide Smilax papyracea.

Um médico de um hospital de comprovada para aguardente referência notificou vários casos de hemorragias gástricas ocorridas com o uso de aguardente alemã. não inflamatória, prurido, dor aguda e calor cutâneo, após a $4^{\mathrm{a}}$ aplicação local de um produto à base de polifenóis de alcachofra.

Uma notificação de ocorrência de taquicardia, em relação a uma formulação adquirida de farmácia de manipulação. A paciente também fazia uso de Passiflora sp. e F. vesiculosus. Este último pode ter exacerbado os sintomas, uma vez que em altas doses este extrato também causa taquicardia.

Uma notificação de taquicardia e hipertireoidismo em relação a uma associação de $F$. vesiculosos, Equisetum arvense, Rhamnus purshiana, sem registro na Anvisa. Uma notificação de interação medicamentosa com Passiflora sp. e E. sinica.

Uma notificação de aplasia medular, infecção garganta, pneumonia e hemorragia, seguida de óbito. A paciente tomava concomitantemente um anticoncepcional.
Uma notificação de inchaço de origem Passiflora sp. e Senna alexandrina,
Há relatos de reações adversas como nervosismo, insônia, taquicardia, extrasístoles, poliúria. As bases xantínicas, sobretudo a cafeína, apresentam uma ação diurética e estimulante do sistema nervoso e cardiorrespiratório. A teofilina tem ação inotrópica positiva. Por seu conteúdo em taninos o extrato etanólico de chá verde pode provocar náuseas e vômitos. $\mathrm{O}$ consumo de C. sinensis foi associado a três casos de desordens hepáticas com elevação de ALT, bilirubina e fosfatase alcalina. Todos os pacientes se recuperaram. Há ainda treze relatos de hepatite em mulheres que tomaram o mesmo extrato por 9 dias a 5 meses.

A ingestão e o contato podem causar sensação de queimação, edema (inchaço) de lábios, boca e língua, náuseas, vômitos, diarréia, salivação abundante, dificuldade de engolir e asfixia; o contato com os olhos pode provocar irritação e lesão da córnea.

Aguardente alemã é uma formulação oficinal composta por uma associação de Operculina macrocarpa e C. scammonia.Há relatos de uso popular, crônico e indiscriminado, para indicações não confirmadas cientificamente.

Pode ter um leve efeito laxativo. É contra-indicado para pessoas com hipersensibilidade a plantas da família Asteraceae. A ocorrência de dermatite de contato alérgica foi relatada.

Pode causar taquicardia. Esta reação adversa pode ser exacerbada pela cafeína, cujo consumo é comum, porém raramente é relatado. Não deve ser administrada por períodos prolongados, uma vez que contém efedrina, cujo consumo é associado a tolerância e dependência.

Em virtude do conteúdo em iodo, supõe-se que estimule a glândula tireóide, aumentando o metabolismo basal. A sobredosagem pode levar a hipertireoidismo, taquicardia e hipertensão.

Há um número crescente de relatos Lobb, 2009. de hepatoxicidade.
Anvisa, 2004b;

Brinker, 2009

Pedros et al., 2003; Vial et al., 2003.

Sinitox, 2009

Fonteles et al., 2008.

Anvisa, 2004b.

Anvisa, 2004b; Brinker, 2009.

Anvisa, 2004b. 


\begin{tabular}{lll}
\hline Ginkgo biloba & $\begin{array}{l}\text { Tratamento de sintomas } \\
\text { da deficiência do fluxo }\end{array}$ & $\begin{array}{l}\text { Três notificações de inefetividade, } \\
\text { sem especificar para qual indicação o }\end{array}$ \\
sanguíneo cerebral: & medicamento estavam sendo tomadas. \\
problemas de memória, & Como popularmente o medicamento \\
cefaléias, vertigem e & é utilizado para estimular a memória \\
distúrbios circulatórios & e a concentração, sem redução do \\
periféricos. & fluxo sanguíneo cerebral associado, a \\
& inefetividade pode estar relacionada \\
& a essa falsa expectativa. Vendido sob \\
& prescrição médica. Duas notificações \\
& de cefaléia e duas de taquicardia. \\
& Uma notificação de náusea, torpor, \\
& formigamento, sudorese excessiva, \\
& hipotensão, rigidez abdominal.
\end{tabular}

Glycine Max $\quad \mathrm{O}$ extrato rico em isoflavonas é indicado para tratamento de sintoma do climatério. O óleo é indicado como infusão de lipídios para nutrição parenteral. O óleo de G. $\max$ e $P$. gratissima é indicado para artroses.

Hypericum perforatum

Tratamento de estados depressivos. Venda sob prescrição médica.

\section{Luffa operculata \\ Descongestionante nasal tópico.}

\section{Mikania glomerata}

\section{Expectorante e}

broncodilatador. O princípio ativo mais conhecido a cumarina.
Há uma notificação com descrição de irritação nasal, anosmia e cefaléia como reações adversas.

Uma notificação de reação adversa em uma criança de 7 anos que apresentou petéquias após o uso de xarope de M. glomerata. Uma notificação de reação alérgica devido ao uso de uma associação preparada em farmácia de manipulação, de $M$. glomerata com Myroxylon balsamum e Cephaelis ipecacuanha.

Vide Convolvulus scammonia.
Há relatos de distúrbios gastrintestinais, cefaléias, taquicardias, hemorragias e hipotensão.

Após administração por via oral, o extrato pode provocar distúrbios gastrintestinais leves como constipação, flatulência e náusea. Embora raros, há relatos de reações de hipersensibilidade, incluindo febre e calafrios, após a administração parenteral.

Há relatos de reações adversas gastrintestinais, que podem ser minimizadas ao administrar o medicamento após as refeições. $\mathrm{O} H$. perforatum induz a via metabólica do citocromo P450, reduzindo o efeito de alguns medicamentos. $\mathrm{O}$ uso concomitante de antidepressivos inibidores da recaptação de serotonina e inibidores da mono-aminoxidase poderá causar síndrome serotoninérgica.

O Boletim informativo da OMS, alerta para a interação medicamentosa com sibutramina, um inibidor da recaptação de serotonina e norepinefrina, podendo levar à síndrome da sero-tonina, com taquicardia, palpitação, hipertensão, suor profuso e dilatação da pupila. Não é recomendado o uso concomitante de drogas fotossensibilizantes como clorpromazina ou tetraciclina.

Há relatos freqüentes de irritação nasal, epistaxe e anosmia, em conseqüência ao seu uso.

$\mathrm{O}$ uso prolongado ou ingestão de altas doses pode gerar taquicardia, vômitos e quadros diarréicos.

Foram observados quadros hemorrágicos em animais após 100 dias de uso contínuo de extratos de guaco. Não deve ser empregado simultâneamente com anticoagulantes, pois as cumarinas podem antagonizar a vitamina $\mathrm{K} \mathrm{e}$ potencializar seus efeitos.

Vide Convolvulus scammonia.
Anvisa, 2004b.

Kaari et

al., 2006;

Tsouronis,

2001;

Martindale,

1996; Maheu et al., 1998.

Anvisa, 2004b; Anvisa, 2004b; Anvisa, 2009b;

Menon-

Miyake et al., 2005.

Anvisa, 2004b.

Fonteles et al 2008. 


\begin{tabular}{|c|c|c|c|}
\hline Panax ginseng & $\begin{array}{l}\text { Adaptógeno, indicado para } \\
\text { estados de fadiga física e } \\
\text { mental. }\end{array}$ & $\begin{array}{l}\text { Quatro notificações de reações adversas } \\
\text { que relatam cefaléia, diarréia e vômitos. }\end{array}$ & $\begin{array}{l}\text { Há relatos de que o uso } \\
\text { prolongado pode provocar insônia, } \\
\text { sangramento nasal, hipertensão, } \\
\text { cefaléia, nervosismo e vômitos, } \\
\text { mastalgias e sangramento vaginal. } \\
\text { Em casos de sobredosagem foram } \\
\text { relatadas arterites cerebrais com } \\
\text { dor de cabeça intensa, diarréia } \\
\text { matinal, náusea e vômito. }\end{array}$ \\
\hline
\end{tabular}

Passiflora Propriedades sedativas. incaranta

Passiflora sp.

A tradição atribui propriedades sedativas, antiespasmódicas e ansiolíticas, parcialmente confirmadas por estudos pré-clínicos.

Pelargonium sidoides

\section{Piper} methysticum

Tratamento da insônia e da ansiedade. agudas e crônicas do trato respiratório. intestinal.

Tratamento sintomático da

Pygeum africanum hiperplasia prostática. cólicas e dormência membros superiores em relação a uma associação de P. incarnata, Salix alba e Crataegus oxycantha.

Uma notificação de taquicardia e hipertireioidismo em relação a um produto manipulado contendo Passiflora sp, Fucus vesiculosus, Rhamnus purshiana, Senna alexandrina e Equisetum arvense. Uma notificação de taquicardia e mal estar de um paciente que tomava Passiflora sp., Fucus vesiculosus, Ephedra sinica e vitaminas. Uma notificação de hepatite fulminante e óbito de um paciente que tomava Passiflora sp., Piper methysticum e vitaminas. Uma notificação de diarréia, flatulência, perda de apetite em relação a um medicamento composto de P. alata, Adonis vernalis, Erythrina mulungu e Leptolobio elegans. Uma notificação de edema e irritação cutânea, com o uso tópico de um creme contendo flavonóides de Passiflora sp.

Uma notificação de obstipação e oligúria. O paciente usava concomitantemente um xarope de Hedera helix.

Uma notificação de hepatite tóxica. Uma notificação de hepatite fulminante, levando a óbito. O paciente tomava concomitantemente Passiflora sp. e vitaminas. Uma notificação de taquicardia, mal estar geral, agitação, sudorese excessiva e cefaléia. $\mathrm{O}$ paciente tomava concomitantemente omeprazol.

Uma notificação de inefetividade.

A reação adversa mais comum é torpor, mas há relatos de reações alérgicas, náusea, vômito e taquicardia severos. Um relato de falência hepática aguda, seguida de morte de uma paciente que tomava uma associação de Piper methysticum, P. incarnata e Scutellaria lateriflora.

Raramente podem ocorrer reações alérgicas.

Anvisa, 2004b Romanini, 2006.

Relatos de reações alérgicas.

Matthys et al., 2003; Boer et al., 2007.

Relatos de hepatotoxicidade, levando à proibição do seu uso em muitos países. Clinicamente, o espectro dessas alterações variou de elevações transitórias das enzimas hepáticas, até à falência hepática fulminante e morte.

Uma notificação de cefaléia. O paciente usava concomitantemente fumarato de rupatadina.
Fisher et al., 2000;

Anvisa, 2004b
Brinker, 2009

Anvisa, 2004b; Anvisa, 2009b; Amorim et al, 2007.
Relatos de flatulência e dor abdominal. Raramente podem ocorrer reações alérgicas.

Distúrbios gastrintestinais leves

Anvisa, 2004b. podem ocorrer. 


\begin{tabular}{|c|c|c|c|c|}
\hline $\begin{array}{l}\text { Senna } \\
\text { alexandrina }\end{array}$ & Laxativo estimulante. & Uma notificação de melanose. & $\begin{array}{l}\text { O extrato não deve ser utilizado } \\
\text { por mais de duas semanas sem } \\
\text { orientação médica, uma vez } \\
\text { que o uso prolongado pode } \\
\text { levar a tolerância, com eficácia } \\
\text { diminuída e necessidade de doses } \\
\text { aumentadas. O uso prolongado } \\
\text { de sene pode levar a inflamação } \\
\text { crônica da mucosa, com acúmulo } \\
\text { de pigmentos melânicos em } \\
\text { leucócitos e apoptose de células do } \\
\text { cólon. As lesões pigmentadas de } \\
\text { pseudomelanose coli desaparecem } \\
\text { com a interrupção do tratamento. }\end{array}$ & Anvisa, $2004 b$. \\
\hline $\begin{array}{l}\text { Smilax } \\
\text { papyracea }\end{array}$ & $\begin{array}{l}\text { Uso tradicional como } \\
\text { depurativo, tratamento } \\
\text { de erupções cutânea e } \\
\text { reumatismo. }\end{array}$ & $\begin{array}{l}\text { Uma notificação de febre, icterícia, } \\
\text { cefaléia, náusea, vômito e tontura, } \\
\text { após o uso de uma associação de } S \text {. } \\
\text { papyracea e Colocasia antiquorum. } \\
\text { Uma notificação de urticária, após uso } \\
\text { de uma associação de } S \text {. papiracea, } \\
\text { Tiamina, Jacaranda caroba e } \\
\text { Trianosperma tayuya. }\end{array}$ & $\begin{array}{l}\text { Relatos de distúrbios } \\
\text { gastrintestinais quando utilizado } \\
\text { em altas doses. }\end{array}$ & Anvisa, 2004b. \\
\hline $\begin{array}{l}\text { Thuja } \\
\text { occidentalis }\end{array}$ & $\begin{array}{l}\text { Uso tópico para tratamento } \\
\text { de verrugas, papilomas e } \\
\text { condilomas. }\end{array}$ & $\begin{array}{l}\text { Uma notificação de ardência, } \\
\text { inflamação da mucosa e cervicite, } \\
\text { após o uso de creme vaginal. Não há } \\
\text { medicamentos fitoterápicos registrados. }\end{array}$ & $\begin{array}{l}\text { O uso oral não é recomendado e } \\
\text { deve ser evitado no período da } \\
\text { amamentação devido à toxicidade } \\
\text { do óleo essencial. }\end{array}$ & $\begin{array}{l}\text { Anvisa, 2004b; } \\
\text { Brinker, } 2009 .\end{array}$ \\
\hline $\begin{array}{l}\text { Tribulus } \\
\text { terrestris }\end{array}$ & $\begin{array}{l}\text { Regulador hormonal } \\
\text { e estimulante da } \\
\text { espermatogênese. }\end{array}$ & $\begin{array}{l}\text { Uma notificação de inefetividade para } \\
\text { tratamento de ejaculação precoce, com } \\
\text { um produto sem registro. }\end{array}$ & $\begin{array}{l}\text { Devido ao teor de saponinas } \\
\text { não deve ser usado por } \\
\text { períodos prolongados. Usado } \\
\text { tradicionalmente na China para } \\
\text { tratamento da ejaculação precoce. }\end{array}$ & Anvisa, $2004 b$. \\
\hline $\begin{array}{l}\text { Uncaria } \\
\text { tomentosa }\end{array}$ & $\begin{array}{l}\text { Tratamento sintomático de } \\
\text { artrites e reumatismo. }\end{array}$ & $\begin{array}{l}\text { Uma notificação de prurido como } \\
\text { reação adversa, em que } U \text {. tomentosa } \\
\text { consta como principal suspeito. }\end{array}$ & $\begin{array}{l}\text { Há relatos febre, diarréia, } \\
\text { constipação, indigestão, } \\
\text { linfocitose, eritrocitose e } \\
\text { agravamento da acne. Pode inibir a } \\
\text { atividade da citocromo P450. }\end{array}$ & Anvisa, 2004b. \\
\hline
\end{tabular}

\section{REFERÊNCIAS}

Amorim M, Diniz M, Araújo M, Pita J, Dantas J, Ramalho J, Xavier A, Palomaro T, Júnior NLB 2007. O papel controvertido da kava (Piper methysticum) - Fitoterápico ansiolítico, na hepatite tóxica. Rev Bras Farmacogn 17: 448-454.

Anvisa 2002a. Resolução no. 356, de 28 de fevereiro de 2002. Alerta UFARM Piper methysticum. Diário Oficial da União.

Anvisa 2002b. Resolução no. 357 de 28 de fevereiro de 2002. Alerta UFARM Hypericum perforatum. Diário Oficial da União.

Anvisa 2004a. Resolução de Diretoria Colegiada no. 48 de 16 de março de 2004. Aprova o regulamento técnico de medicamentos fitoterápico junto ao SNVS. Diário Oficial da União.

Anvisa 2004b. Resolução no. 88 de 16 de março de 2004. Dispõe sobre a Lista de referências bibliográficas para avaliação de segurança e eficácia de fitoterápicos. Diário Oficial da União.

Anvisa 2009a. Resolução no. 4 de 10 de fevereiro de 2009. Dispõe sobre as normas de farmacovigilância para os detentores de registro de medicamentos de uso humano.
Diário Oficial da União.

Anvisa 2009b. Resolução no. 47 de 8 de setembro de 2009. Dispõe sobre as regras para elaboração de bulas de medicamentos. Diário Oficial da União.

Anvisa 2009c. Onovo conceito da farmacovigilancia. Disponível em:http://www.anvisa.gov.br/farmacovigilancia/ apresenta.htm. Acesso agosto 2009.

Bames J 2003. Pharmacovigilance of herbal medicines: a UK perspective. Drug Saf 26: 829-851.

Boer HJ, Hagemann U, Bate J, Meyboom RH 2007. Allergic reactions to medicines derived from Pelargonium species. Drug Saf 30: 677-680.

Brinker F 2009. Herb contraindications and drug interactions, 3rd ed. Sandy, Oregon: Eclectic Medical Publications.

Capasso R, Izzo AA, Pinto L, Bifulco T, Vitobello C, Mascolo N 2000. Phytotherapy and quality of herbal medicines. Fitoterapia 71: 58-65.

Council for International Organizations of Medical Sciences 2001. Current Challenges in Pharmacovigilance:Pragmatic Approaches. Report of CIOMS Working Group V, 381p.

Congresso Nacional 1973. Lei $n^{\circ} 5991$, de 17 de dezembro de 1973. Dispõe sobre o controle sanitário do comércio de drogas, medicamentos, insumos farmacêuticos e 
correlatos, e dá outras providências.

Cupp MJ 1999. Herbal remedies: adverse effects and drug interactions. Am Fam Physicians 59: 1239-1245.

Fisher AA, Purcell P, Le Couteur DG 2000. Toxicity of Passiflora incarnata L. J Toxicol Clin Toxicol 38: 63-66.

Fonteles M, Venâncio E, Rios VR, Bessa MB, Francelino E, Carvalho D, Coelho H 2008. Vigilância póscomercialização da aguardente Alemã ${ }^{\circledR}(O$. macrocarpa e C. scammonea). Rev Bras Farmacogn 18: 748-753.

Kaari C, Haidar MA, Soares Júnior JM, Nunes MG, Quadros LG, Kemp C 2006. Randomized clinical trial comparing conjugated equine estrogens and isoflavones in postmenopausal women: a pilot study. Maturitas 53:4958 .

Lobb A 2009. Hepatoxicity associated with weight-loss supplements: A case for better post-marketing surveillance. World J Gastroenterol 15: 1786-1787.

Maheu E, Mazières B, Valat JP, Loyau G, Le Loet X, Bourgeois P, Grouin JM, Rozenberg S 1998. Symptomatic efficacy of avocado/soybean unsaponifiables in treatment of osteoarthritis of the knee and hip. Arthritis Rheum 41: 81-91.

Matthys H Eisebitt R, Seith B, Heger M 2003. Efficacy and safety of an extract of Pelargonium sidoides (EPs 7630) in adults with acute bronchitis. Phytomedicine 10: 7-17.

Martindale - The Extra Pharmacopoeia 1996. Royal Pharmaceutical Society. 31 ed. London, 493 p.

Mendes MC, Pinheiro R, Avelar K, Teixeira J, Silva GS 2008. História da farmacovigilância no Brasil. Rev Bras Farm 89: 246-251.

Menon-Miyake M, Saldiva P, Lorenzi-Filho G, Ferreira M, Butugan O, Oliveira R 2005. Efeitos da Luffa operculata sobre o epitélio do palato de rã: aspectos histológicos. Rev Bras Otorrinolar 71: 132-138.

Ministério da Saúde 2006. Portaria no. 971, de 3 de maio de 2006. Aprova a Política Nacional de Práticas Integrativas e Complementares (PNPIC) no SUS. Diário Oficial da União.

Pedros C, Cereza G, Garcia N, Laporte N 2003. Liver toxicity of Camellia sinensis dried ethanolic extract. Med Clin 121: 598-599.

Romanini C 2006. Avaliação da atividade ansiolítica e antidepressiva do extrato fluido e fração aquosa de folhas de Passiflora alata Curtis em camundongos. Acta Sci 28: 159-159.

Sinitox 2009. Sistema Nacional de Informações Tóxicofarmacológicas. http://www.fiocruz.br/sinitox/ taiobabrava.htm. Acesso em setembro 2009.

Tsouronis C 2001. Clinical effects of phytoestrogens. Clin Obstet Gynecol 44: 836-842.

Veiga Junior V, Pinto A, Maciel MA 2005. Plantas medicinais: cura segura? Quim Nova 28: 519-528.

Vial T, Bernard G, Lewden B, Descotes J 2003. Acute hepatitis due to exolise, a Camellia sinensis-derived drug. Gastroenterol Clin Bio 27: 1166-1167.
World Health Organization 2002. The importance of Pharmacovigilance - Safety Monitoring of Medicinal Products. Geneva.

World Health Organization 2004. WHO Guidelines on safety monitoring of herbal medicines in pharmacovigilance systems. Geneva.

Wiffen PJ, Gill M, Edwards J, Moore A 2002. Adverse drug reactions in hospital patients: a systematic review of the prospective and retrospective studies. Bandolier Extra June: 1-14. 